\title{
The diamond cutting technology in hydrotechnical concrete structures
}

\author{
Artem Subbotin ${ }^{1, *}$ and Daniil Morev ${ }^{1}$ \\ ${ }^{1}$ Moscow State University of Civil Engineering, Yaroslavskoe shosse, 26, Moscow, 129337, Russia
}

\begin{abstract}
The possibility of improving the performance of diamond cutting under water is a relevant research not only for the construction industry, but also in the field of environmental protection and the environmental situation in the area of hydraulic structures. The objects of the study are hydraulic structures, namely reinforced concrete and concrete structural elements in contact with water. The objectives of the study are to analyse the methods of diamond cutting and drilling of structures, compare Russian and international experience in the production of these types of work, assess the environmental impact of cutting and drilling structures in water, and offer an effective organizational form for managing and systematizing diamond operators cutting. The basis of the research methodology is the study of the works of domestic and foreign authors in the field of organization of construction and installation works, their technology and mechanization. During the work on the topic, the authors reviewed and compiled the experience of international and Russian results of theoretical and applied research in the development and improvement of the organization theory and technology for the reconstruction of concrete and reinforced concrete structures of buildings and structures that made a huge contribution to the development of diamond cutting technologies.
\end{abstract}

\section{Introduction}

At the moment, increasing production efficiency in any industry is a very important task, since such indicators as the quality of production, the speed of work, and the economic and environmental components of these issues depend on efficiency.

Thus, the relevance of scientific work is to address the promising issues of improving the efficiency of diamond cutting technologies and drilling hydraulic structures that arise during the reconstruction, which takes into account the high requirements for compliance with environmental standards. It is also important to note that the increase in the efficiency of any production, in particular, the technology of diamond cutting of hydraulic structures, makes it possible to save both labor and material costs. The object of research of this work will be the technology of diamond cutting of hydraulic structures with suggestions for improving its efficiency.

This article discusses the key research objectives:

\footnotetext{
*Corresponding author: subbotin-art@mail.ru
} 
- reviewing the experience of international and Russian scientists in the field of technology and the organization of construction, as well as environmental protection;

-to analyze the various methods of dismantling used in the reconstruction of hydropower plants;

- indicate the reasons for reducing the negative impact on the environment.

- identify proposals for improving the efficiency of the diamond tool.

In our time, the question of the reconstruction of buildings and structures, in particular, the reconstruction of unique structures, for example, hydraulic facilities (concrete spillway dams, dams, locks, etc.), is becoming increasingly common.

The purpose of the study is to study the possibility of improving the efficiency of diamond cutting and drilling in the conditions of hydraulic structures.

In the course of work on the topic, the results of applied and theoretical studies of Russian and foreign scientists, who made a great contribution to the development and improvement of the theory, methodology of organization and technology for the construction of concrete and reinforced concrete structures below the water level were studied. The basis of diamond technology is the process of cutting concrete and reinforcement with diamond segments mounted on the tool body (crowns, discs, ropes, etc.). The tool is driven by a specialized wall saw or drilling machine.

\section{Scientific work}

The work deals specifically with hydropower facilities, since they must meet the requirements of special increased responsibility, both during construction and during reconstruction. Also, to hydraulic structures put forward increased standards for environmental friendliness, due to the fact that these objects are in contact with water.

Hydrotechnical structures are constantly subject to dynamic influence from the water side - waves and water discharge from the upstream to the lower one, because of this there is a deterioration of concrete, which must be dismantled. For this it is necessary to resort to special methods and technologies. These methods include:

a) drilling and blasting method, which consists in the use of explosives in the zone of dismantling;

b) concrete crushing by pneumatic hammers;

c) the use of special chemical reagents injected into pre-drilled wells.

Recently, the diamond cutting method has been increasingly used in the reconstruction of hydraulic structures (locks, spillways, etc.).

The advantage of any kind of technology over others today is determined by how it satisfies technological and environmental standards. The positive aspects of this method are quite weighty. For example, the high precision of dismantling allows you to reconstruct objects of increased responsibility, such as hydroelectric power plants, where dismantling needs to be performed under limited tolerances. Also, this technology allows you to save a large amount of time for disassembly than with explosive and shock methods, in which there is a strong scatter of the elements to be dismantled, since crushing occurs on different parts of different sizes. The relatively low level of noise (an average of $86 \mathrm{~dB}$ ) and vibrations, which affects the psychological and physical effects on people, as well as reduces the risk of the formation of occupational diseases. Diamond cutting technology is environmentally friendly and preferred in terms of water, water, water, water and water. The above positions affect the speed of work, which is a strong factor in choosing the method of diamond technology. In addition, the technology of diamond cutting allows solving complex problems, for example, the total dismantling of reinforcement and concrete, which is impossible when using other methods. Data on the availability of 
securities as a percentage of other methods is given above, which is the main factor in choosing this research topic.

In confirmation of this, several examples are presented using diamond cutting technologies in the reconstruction of hydraulic structures. For example, the reconstruction of the Folslm dam (California, USA) in 1998- Figure 1, the manufacture of large-diameter holes at the Akulovsky hydroelectric complex of Mosvodokanal, the reconstruction of the Novorossiysk seaport - the repair of an oil pier, the dismantling of a part of the mooring wall of the Yakhroma hydroelectric complex and many others.

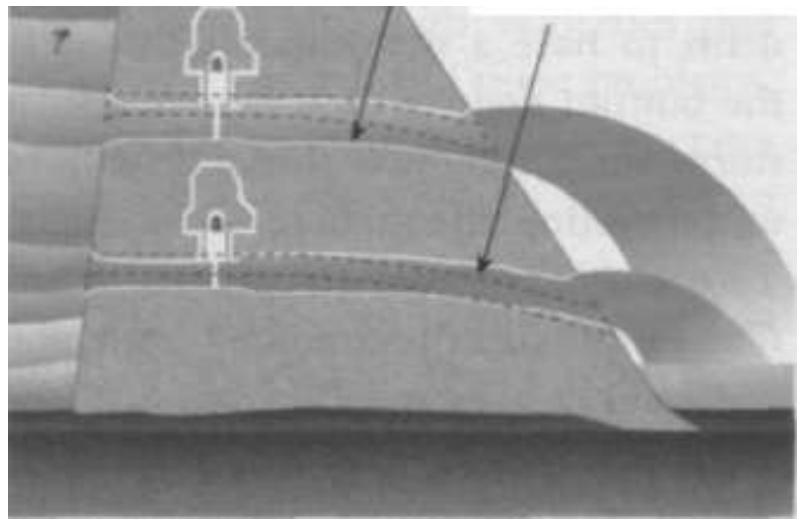

Fig. 1 Change in geometric parameters of the Folsom dam water lines.

Quite often, the technology of diamond cutting, when other methods of dismantling are not feasible, for example, by court to find parts of structures under water. Accordingly, the corresponding statement, it is necessary to propose methods for improving the efficiency of diamond tools, since the frequency of using this technology is becoming popular.

The technology of diamond cutting has become a popular technology that allows orienting diamond grains in a bundle. There are many ways to manufacture artificial diamonds, for example, HPHT (high pressure and high temperature method) or CVD (with the addition of hydrogen). This has led to increased interest in the study of industrial diamond in various fields from electrical engineering to construction.

The effectiveness of the work depends on many factors that indirectly and directly affect the process and economic feasibility. Development and use of diamond tools. Currently, there are various types of machines for diamond work: for drilling, for cutting, grinding and many others. 1 The block diagram of the classification of the main types of diamond technology in Figure 2.

In the reconstruction of hydraulic structures, rope and diamond disc tools are often used. For each of them you need to choose your mode of operation. The right choice of tools will lead to increased efficiency of the work performed

The use of diamond equipment is very diverse, and most importantly mobile. Work can be performed in any position, to correct the process of destruction and to dismantle any form.

As already indicated above, hydraulic structures are of the first class of responsibility. Therefore, for each of the objects it is necessary to develop a special project for the implementation of works. In practice, the project preparation time is up to $80 \%$ of the total period of work. The process of dismantling itself for structures under water is remote, which significantly reduces the period of work due to the lack of training activities for specialists' divers. Also in labor protection it is important that a worker be in a zone of safe activity in order to reduce risks, in our case due to the fact that work is performed remotely, the number of risks decreases several times. 


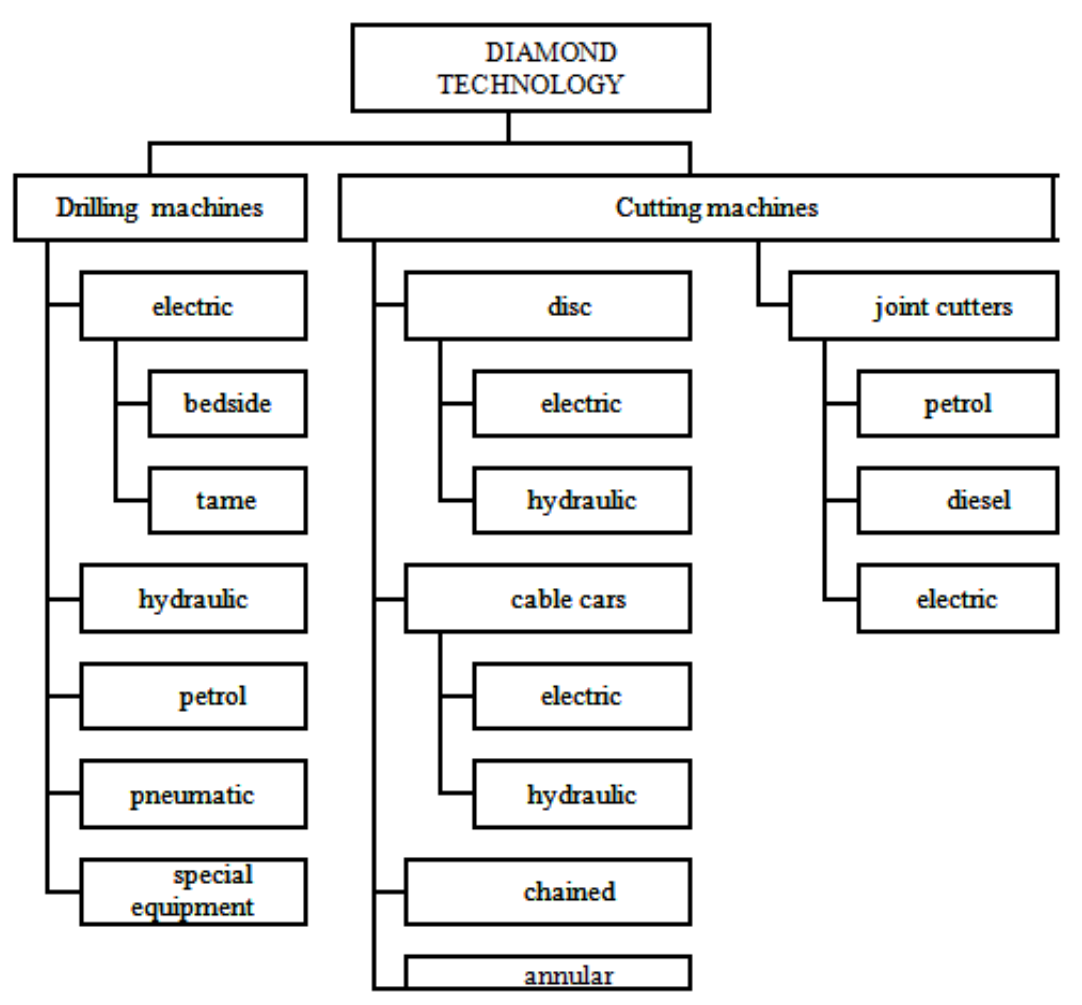

Fig. 2. Block diagram of the classification of the main types of diamond technology.

As mentioned above, the mode of operation depends on the properties of the medium. Since hydraulic structures are mainly in contact with water, it is necessary to take into account its physical, chemical and mechanical properties. For example, when taking into account the chemical properties of water, which acts as a dipole molecule, falling into a magnetic field created by a diamond tool, there is a decrease in tool wear due to the orientation of the water dipole along the surface of the diamond bundle, creating a layer of non-liquid water and thereby reducing tool wear. Another example is that water reduces the strength of concrete and makes it plastic, as far as possible for a brittle material, and the mode of operation from microcutting to relatively plastic extrusion of sludge with a diamond tool changes accordingly. For this fact, it is necessary to choose a rational technology of work that will increase the efficiency of work due to the reduction in wear of the diamond ligament. Since the density of water is a thousand times higher than the density of air, the rate of removal of sludge from the cutting zone is several times reduced, it follows that it is necessary to work to remove the sludge from the cutting zone, otherwise the tool will slide on the concrete surface and not cut. This problem is solved by increasing the water supply to the cutting zone and washing out the sludge from there.

Selecting the correct cutting mode, you need to know the production technology of work, for example, increasing the speed of rotation of the tool increases its durability and, as a result, wear decreases.

As practice shows, the technology of organization of construction production, mode of operation rather strongly affects the efficiency of the work performed. Mode of operation depends on the operator of the machine. Work efficiency will increase due to the competence and qualifications of the operator. This dependency can be continued by creating regulatory documentation. 
Factors affecting the increase in work efficiency are presented in Figure 3.

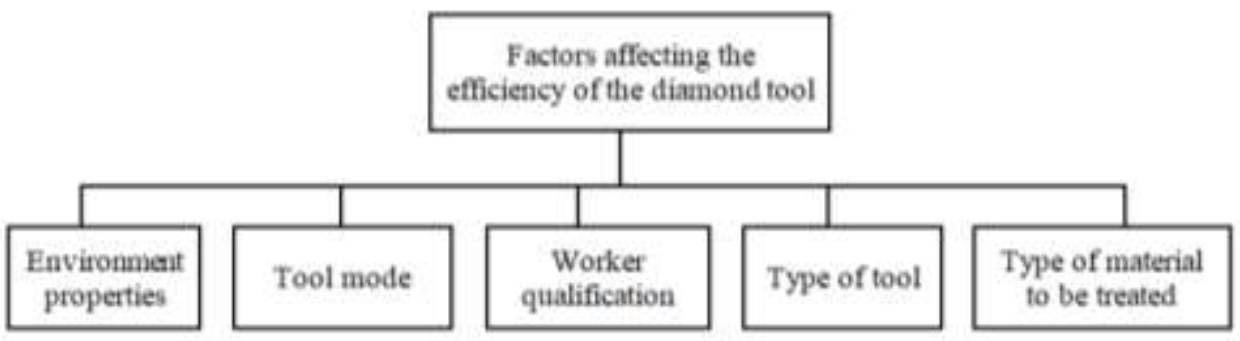

Fig. 3. Block diagram of the classification of factors affecting the efficiency of the diamond tool.

From all the above, the following possibilities to improve the efficiency of diamond cutting of hydraulic structures can be highlighted:

1. To increase the intensity of leaching of sludge during cutting, by increasing the water supply to the cutting zone;

2. Training and advanced training of specialists in concrete machining with diamond tools under water;

3. Selection of the right tool for diamond cutting of hydraulic structures will lead to the speed of work and, as a consequence, to increase efficiency, for example, to reduce the thickness of the diamond tool cross-section, so that turbulent twists act with less force;

4. Accounting for the physical, mechanical and chemical properties of the material being processed will increase efficiency if the variability of the properties of the material in the preparation of a special project is introduced.

\section{Conclusion}

Thus, improvement of the efficiency of diamond cutting leads to a reduction in costs due to a decrease in wear of the diamond tool. Also, the lack of additional work on grinding the surface and dismantling construction debris from dismantled structures, which leads to an increase in the speed of dismantling. This method meets water use and environmental regulations.

The following are the advantages of diamond cutting technology compared to other technologies:

1. The absence of shock, wave and vibration loads on structures;

2. High precision dismantling without the need for further surface treatment;

3. The absence of dust and debris and, as a consequence, the absence of a negative impact on the environment;

4. Works are performed without stopping the existing production;

5. Safe cutting of supporting structures;

6. High speed of work at low labor and energy costs;

7. Unlimited thickness and depth of the structure;

8. Low storage costs;

9. High mobility of equipment;

10. Fast solution of complex tasks.

The paper presents the factors that directly affect the possibility of improving the efficiency of the diamond cutting method when working under water. These factors include:

1. Selection of the correct mode of operation - cutting speed, cutting depth, and more;

2. Backing the right tool and reducing its cross-section from all possible types, it is preferable to use the method of diamond wire cutting; 
3. Consideration of the physical, chemical and mechanical properties of the medium and the material being processed, which must be included in the reconstruction project to achieve accuracy and speed of work performed.

\section{References}

1. Technical Data, International document (München, 2007)

2. Tolerance and Limits for Construction Drilling and Sawing, International document (Zurich, 2005)

3. B.V. Zhadanovsky, PhD Thesis (Moscow, 2018)

4. M.G. Zertsalov, A.V. Kosolapov, Abstracts of the report at the conference «Hydropower. New developments and technologies» (St. Petersburg, 2010)

5. A.V. Kosolapov, M.G. Zercalov, National Association of Diamond Cutting and Drilling 3, 12-17 (2014)

6. A.V. Kosolapov, The use of technology of diamond cutting and drilling in the repair and reconstruction of hydraulic structures, Dissertation (Moscow, 2010)

7. V.Yu. Sadovoy, The study of the secondary electron emission of a single crystal diamond, Dissertation (Dolgoprudny, 2016)

8. V.Yu. Komarovskikh, The study of the structure and electronic state of paramagnetic centers in diamond, associated with the entry of phosphorus, oxygen, hydrogen, silicon and germanium (Novosibirsk, 2016)

9. A.N. Pushkin, N.N. Zenin, O.K. Gulish, O.A. Kuznetsova, A.P. Rudenko, Journal of Physical Chemistry, Nauka Publishing House (M) 73(9), 1561-1566 (2009)

10. A.V. Kosolapov, Theses of the report at the International Atomic Forum "Bulatom" (Varna, Bulgaria, 2008) 